\title{
A Solution to the Bundle-to-Bundle Mapping Problem of Geometric Optics Using Four Freeform Reflectors
}

\author{
Christopher B. Croke ${ }^{1, *}$ and R. Andrew Hicks ${ }^{2}$ \\ ${ }^{1}$ Department of Mathematics, University of Pennsylvania, Philadelphia PA 19104 \\ ${ }^{2}$ Department of Mathematics, Drexel University, Philadelphia PA 19104
}

\begin{abstract}
Here we present a method for the coupled design of four free-form reflective surfaces which will control a bundle of rays. By this we mean that given an input bundle of rays, we can construct an optical system that will map it to a given output bundle, where a ray to ray correspondence is realized as per the prescribed data. The method makes use of the Cartan-Kähler theorem of Exterior Differential Systems. Sample imaging applications are given.

OCIS codes: $\quad 080.2740,110.0110,230.4040$.

http://dx.doi.org/10.1364/XX.99.099999
\end{abstract}

\footnotetext{
* Corresponding author: ahicks@drexel.edu
} 


\section{Introduction}

We show here how to entirely control a single 2-parameter bundle of rays, in the sense that given a target point on a surface assigned to each ray and a desired incident direction at each such point, we can from this prescribed data design a system of four reflectors which will optically realize the required correspondence. Put another way, we solve a basic problem of geometric optics, namely the construction of optical system that maps a given input ray bundle to a given output bundle, where each input ray is mapped to a pre-determined output ray. We not only achieve a "shape to shape correspondence" between the bundles but also realize a given correspondence at the ray level. This allows for a larger class of applications, especially in the realm of imaging.

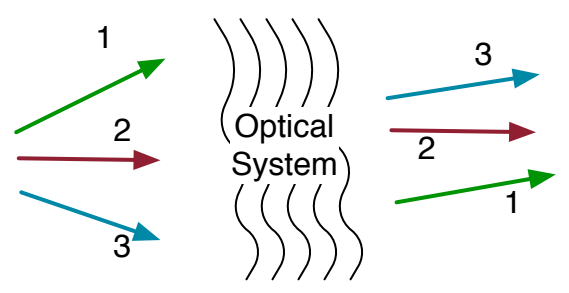

Fig. 1. A schematic description of our problem - we wish to design an optical system consisting of mirrors that maps a ray bundle to a ray bundle on the ray level.

Note that we do not assume any symmetry in the problem, which results in the solution surfaces typically being freeform, i.e. those surfaces which are not a portion of a surface of revolution. Additionally, without any assumptions of symmetry, it follows from the theory of Exterior Differential Systems(EDS) that generically four surfaces is the exact number needed to solve this problem.

Because we live in a golden age of fabrication, we also live in a golden age of optical design. Problems that were previously not considered are now being examined with the result that considerable interaction is taking place between multiple fields of mathematics and optics. We will describe our viewpoint of optical design and delve briefly into the background below.

The fundamental problem of design in geometric optics is to control collections of light rays. The light rays typically consist of bundles emanating from a collection of point sources, with two parameters describing the rays emanating from each source. (One may consider one-parameter families, or even a finite number of rays mixed in, but these problems are 
less common.) Extended sources may be approximated using multiple point sources. There are at least two different manners in which one might want to control the rays emanating from a single source.

In the first, the designer could be given a collection of prescribed target points, with one target point assigned to each ray in the bundle, with the design problem then being to produce an optical system through which each ray will pass and be directed to its prescribed target point. When each individual ray is given a prescribed target point we refer to this as an imaging problem. Generally the target points form a surface, which is usually a plane, (but not always) and we refer to the associated correspondence between rays and target points as the projection and the problem as the prescribed projection problem. It is the fundamental problem of imaging. If there is one target point for each source, it is the fundamental problem of photography.

In the second problem the goal is to achieve a bundle to bundle mapping, but without having to follow a prescribed correspondence on the ray level. Thus, what the rays in the interior of the bundle are doing is not of interest. In other words, the goal is to transform the shape of the bundle into a prescribed shape, in a prescribed position.

The problem of interest in this paper is a combination of the first problem and second problem. In addition to requiring that the output rays strike assigned target points, they must strike their target points with prescribed incoming directions. This problem is thus the general problem of mapping a given ray bundle in image space to an output ray bundle in object space, but with a requirement on where the individual rays go. Put yet another way, given an input bundle we require that the output bundle have a prescribed shape, and that additionally the "internal assignment" of rays also follow a prescription. We view this as an imaging problem because the control of individual rays is required. We test our designs quite convincingly in the graphical ray tracing package POVRay.

The prescribed projection problem was first described by the second author and R. Perline in 2000 [1] (although rotationally symmetric forms had been considered for laser beam shaping e.g. $[2,3])$. In this case the problem under consideration was to use a single mirror to solve the single source problem and it was shown that this was equivalent to the mathematical problem of integrating an over-constrained pair of partial differential equations. A sample application of this problem is the design of a driver-side mirror for a motor vehicle, which has no blind-spot and yet provides the observer with a perspective view of the scene $[1,4,5]$. 
Note that this is an imaging case of the problem which is not photographic, i.e. the ray bundle is not mapped to a single point but spread out on a plane. This work centers on finding approximate solutions for the prescribed projection problem in the case of a single source, since a single reflector generically does not suffice to solve this problem.

If one has a single source and two reflectors the problem is generally solvable, as was shown by the authors in [6] using ideas from EDS. That work is the main predecessor of this work.

A more demanding variant of the second problem is to prescribe the energy intensity on a target surface. Then the rays must be guided to this surface from the source, but where individual rays end up is still of no concern - one simply wants the total energy to match the prescribed intensity. (Natural terminology, although not yet universal, would be to refer to this as the prescribed intensity problem). Interestingly, the single source problem in the illumination case was also not given a precise mathematical form and solved exactly until the late 1990s by Oliker et al in $[7,8]$. An interesting earlier analysis on this problem was done by Schruben in 1972 [9].

All of these problems, and in particular the one of interest in this paper, are linked to the fabrication and design of freeform surfaces. Historically, of course, it has been almost impossible to machine freeform surface to optical quality. With the appearance of computer controlled machining, it became possible to make a surface with any almost any profile on a lathe. Early freeform designs include a progressive spectacle lens designed in the late 1950s by Kanolt [10] and the Polaroid SX-70 folding camera [11] whose key components were mostly patented in the early 1970 s, e.g. [12-14].

Only in the last fifteen years has technology existed that can produce optical quality freeform surfaces. This technology was for the most part developed as part of the Defense Advanced Research Projects Agency (DARPA) conformal optics program [15, 16]. Since it was never possible to fabricate these surfaces until recently, little design theory was ever developed; exceptions include $[17,18]$.

The work of Oliker et al on illumination is spiritually parallel to the problems considered by the authors in that it describes the fundamental mathematical theory of a basic illumination problem. Other fundamental theory of illumination includes that of Rubinstein and Wolansky in [19], which describes a means of designing freeform lenses to control the intensity of a collimated beam. 
The reader should bear in mind though that the general engineering problem of designing systems for illumination has a vast literature, which is well surveyed in [20]. In general the problem of controlling multiple bundles simultaneously is unsolved, as is discussed in [20]. In particular the edge-ray principle is closely related to the problem of preserving bundle shape. A recent and popular application of illumination optics has been due to the widespread use of light emitting diodes. Note that illumination design has applications to areas such as laser beam shaping [21] and solar collector design [22]. Regarding this last reference, much of this work was heavily motivated by the interest in the 1970s in solar collectors, and a classic work describing the state of the art circa 1978 is by Welford et al in [23].

\section{The Design Algorithm}

The mathematical theory of EDS developed by Cartan and Kähler [24] implies that four reflectors are required to produce an exact solution to this problem. It also implies that fewer than four will not typically suffice. The theory does not tell us though how to go about constructing solutions to the problem, either in closed form or numerically. That is partly our contribution here.

We model the reflectors as a system of first order partial differential equations. We will label the rays in our bundle with points in the $y-z$ plane (and so it is assumed that no two rays intersect each other in this plane). We will use $(s, t)$ to parameterize the incoming rays so that $R(s, t)$ is the unique ray that passes through the point $(0, s, t)$ in the $y-z$ plane. (In our numerical examples $R(s, t)$ will be parallel to the $x$-axis). This ray will then successively reflect off of four reflectors $M_{1}, M_{2}, M_{3}$, and $M_{4}$ at points $M_{1}(s, t)$, $M_{2}(s, t), M_{3}(s, t)$, and $M_{4}(s, t)$. After the last reflection from $M_{4}$ the ray will coincide with a prescribed ray $R_{\text {out }}(s, t)$. (Thus the target point information and the incident directions are both encoded into $R_{\text {out }}$.) To summarize, our given data is a parameterized incoming ray bundle, $R(s, t)$ and a parameterized outgoing bundle, $R_{\text {out }}(s, t)$, and are asked to determine the four parameterized surfaces (mirrors) $M_{i}(s, t)$ such that each ray $R(s, t)$ will reflect in sequence off the mirrors $M_{i}$ at $M_{i}(s, t)$ and end up as the ray $R_{\text {out }}(s, t)$.

We point out that although our method applies to rather general ray bundles they must satisfy certain conditions. As we mentioned above, the incoming bundle ray bundle must intersect the $x-y$ plane nicely. Similarly we need the outgoing bundle to intersect a smooth 


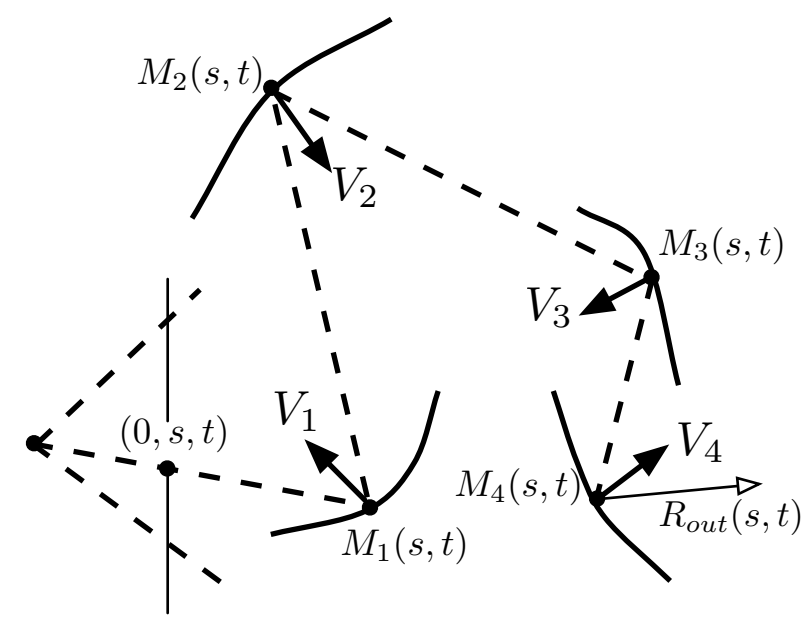

Fig. 2. The surfaces $M_{1}, M_{2}, M_{3}$ and $M_{4}$ must all be perpendicular to the ray path. This imposes conditions on their derivatives.

embedded surface $\mathcal{S}$ nicely so that the induced map from the $x-y$ plane to the surface $\mathcal{S}$ is a diffeomorphism. Note that the fourth mirror might need to be huge for some choices of output bundles.

There is a strong restriction though that the surfaces $M_{i}$ must satisfy. Namely, that each one must be perpendicular to the vector field defined by computing the sum of the incoming and outgoing rays.

The $M_{i}$ are surfaces parameterized by points in the $y-z$ plane:

$$
M_{i}:(s, t) \mapsto\left(x_{i}(s, t), y_{i}(s, t), z_{i}(s, t)\right)
$$

Of course, in order for the final output ray to be $R_{\text {out }}(s, t)$ we will need to satisfy the condition that the point $M_{4}(s, t)$ lie on the ray $R_{\text {out }}(s, t)$. The only other conditions we need are that the surfaces $M_{i}$ are normal to certain vectors $V_{i}$ at the point $M_{i}(s, t)$, which are naturally determined by the ray path (see Fig. 2). We let $V R(s, t)$ be the unit vector at the point $M_{1}(s, t)$ in the direction of the ray $R(s, t)$, and similarly $V R_{\text {out }}(s, t)$ be the unit vector at the point $M_{4}(s, t)$ in the direction of the ray $R_{\text {out }}(s, t)$. Then the $V_{i}$ are as follows:

$$
\begin{gathered}
V_{1}(s, t)=-V R(s, t)+\frac{M_{2}(s, t)-M_{1}(s, t)}{\left|M_{2}(s, t)-M_{1}(s, t)\right|} \\
V_{2}(S, t)=\frac{M_{1}(s, t)-M_{2}(s, t)}{\left|M_{1}(s, t)-M_{2}(s, t)\right|}+\frac{M_{3}(s, t)-M_{2}(s, t)}{\left|M_{3}(s, t)-M_{2}(s, t)\right|}
\end{gathered}
$$




$$
\begin{gathered}
V_{3}(s, t)=\frac{M_{2}(s, t)-M_{3}(s, t)}{\left|M_{2}(s, t)-M_{3}(s, t)\right|}+\frac{M_{4}(s, t)-M_{3}(s, t)}{\left|M_{4}(s, t)-M_{3}(s, t)\right|} \\
V_{4}(s, t)=V R_{\text {out }}(s, t)+\frac{M_{2}(s, t)-M_{1}(s, t)}{\left|M_{3}(s, t)-M_{4}(s, t)\right|} .
\end{gathered}
$$

Thus the problem is to find four parameterized surfaces $M_{i}(s, t)$ that are perpendicular to the vectors $V_{i}(s, t)$, which we will refer to as condition $\mathbf{1}$, and further that $M_{4}(s, t)$ lies on the required ray $R_{\text {out }}(s, t)$, which we will refer to as condition 2 .

It turns out to be useful to put the parameterized surfaces together as a single parameterized surface $\Gamma$ in 12 -dimensional space, $\mathbb{R}^{12}$, with the parametrization

$$
\Gamma(s, t)=\left(x_{1}(s, t), y_{1}(s, t), z_{1}(s, t), x_{2}(s, t), y_{2}(s, t), z_{2}(s, t), x_{3}(s, t), \ldots, z_{4}(s, t)\right)
$$

We emphasize that $\Gamma$ is a 2 -dimensional object in 12 -space whose 2 -dimensional tangent plane is spanned by the vectors $\Gamma_{s}=\partial_{s} \Gamma$ and $\Gamma_{t}=\partial_{t} \Gamma$. Condition 1 requires that for all $(s, t)$ that $\Gamma_{s}$ and $\Gamma_{t}$ are perpendicular to four vector fields $\widetilde{V}_{i}$ in $\mathbb{R}^{12}$ that are derived from the $V_{i}$. To define $\widetilde{V}_{2}$ suppose we are a given 12 -tuple $\left(x_{1}, x_{2}, x_{3}, \ldots x_{12}\right) \in \mathbb{R}^{12}$. Then take $\left(X_{2}, Y_{2}, Z_{2}\right)$ in $\mathbb{R}^{3}$ to be

$$
\left(X_{2}, Y_{2}, Z_{2}\right)=\frac{\left(x_{1}, x_{2}, x_{3}\right)-\left(x_{4}, x_{5}, x_{6}\right)}{\left|\left(x_{1}, x_{2}, x_{3}\right)-\left(x_{4}, x_{5}, x_{6}\right)\right|}+\frac{\left(x_{7}, x_{8}, x_{9}\right)-\left(x_{4}, x_{5}, x_{6}\right)}{\left|\left(x_{7}, x_{8}, x_{9}\right)-\left(x_{4}, x_{5}, x_{6}\right)\right|} .
$$

Then we have

$$
\widetilde{V}_{2}=\left(0,0,0, X_{2}, Y_{2}, Z_{2}, 0,0,0,0,0,0\right)
$$

The vector field $\widetilde{V}_{3}$ is defined similarly.

Since $V_{1}$ and $V_{4}$ depend on the given $R(s, t)$ and $R_{\text {out }}(s, t)$ the vector fields $\widetilde{V}_{1}$ and $\widetilde{V}_{4}$ are a bit more complicated. We first will define functions $s\left(x_{1}, x_{2}, x_{3}\right)$ and $t\left(x_{1}, x_{2}, x_{3}\right)$ as the values of $(s, t)$ such that the ray $R(s, t)$ (that by definition passes through the point $(0, s, t)$ ) passes through the point $\left(x_{1}, x_{2}, x_{3}\right)$. For the situations we will consider we assume that such $(s, t)$ is defined and unique. Now we can define at $\left(x_{1}, x_{2}, x_{3}, \ldots x_{12}\right) \in \mathbb{R}^{12}$

$$
\left(X_{1}, Y_{1}, Z_{1}\right)=-V R\left(s\left(x_{1}, x_{2}, x_{3}\right), t\left(x_{1}, x_{2}, x_{3}\right)\right)+\frac{\left(x_{4}, x_{5}, x_{6}\right)-\left(x_{1}, x_{2}, x_{3}\right)}{\left|\left(x_{4}, x_{5}, x_{6}\right)-\left(x_{1}, x_{2}, x_{3}\right)\right|}
$$

and

$$
\widetilde{V}_{1}=\left(X_{1}, Y_{1}, Z_{1}, 0,0,0,0,0,0,0,0,0\right)
$$


while

$$
\left(X_{4}, Y_{4}, Z_{4}\right)=\frac{\left(x_{7}, x_{8}, x_{9}\right)-\left(x_{10}, x_{11}, x_{12}\right)}{\left|\left(x_{7}, x_{8}, x_{9}\right)-\left(x_{10}, x_{11}, x_{12}\right)\right|}+V R_{\text {out }}\left(s\left(x_{1}, x_{2}, x_{3}\right), t\left(x_{1}, x_{2}, x_{3}\right)\right)
$$

and

$$
\widetilde{V}_{4}=\left(0,0,0,0,0,0,0,0,0, X_{4}, Y_{4}, Z_{4}\right) .
$$

In the examples below the incoming beam is parallel to the $x$-axis and the output beam is (in most cases) collimated in the $(1,1,1)$ direction, and hence things are much simpler since $s\left(x_{1}, x_{2}, x_{3}\right)=x_{2}, t\left(x_{1}, x_{2}, x_{3}\right)=x_{3}, V R(s, t)=(1,0,0)$, and $V R_{\text {out }}(s, t)=\frac{1}{\sqrt{3}}(1,1,1)$ (i.e. $V R$ and $V R_{\text {out }}$ are constant).

Thus our problem becomes to find a surface $\Gamma(s, t)$ in $\mathbb{R}^{12}$ which is everywhere perpendicular to the four vector fields $\widetilde{V}_{i}$ and also satisfies condition 2. Satisfying condition 2 means that the point lies in a subset $\mathcal{M} \subset \mathbb{R}^{12}$ which is a 10-dimensional constraint space. $\left(x_{1}, x_{2}, x_{3}, \ldots x_{12}\right) \in \mathcal{M}$ if the point $\left(x_{10}, x_{11}, x_{12}\right)$ lies on the ray $R_{\text {out }}\left(s\left(x_{1}, x_{2}, x_{3}\right), t\left(x_{1}, x_{2}, x_{3}\right)\right)$. Note that the contraint that a point in 3-space lies on a given ray is in some sense two constraints, which is why the constraint space $\mathcal{M}$ is $12-2=10$ dimensional. Another way to see this 10 dimensionality explicitly is to observe that for each $\left(x_{1}, x_{2}, \ldots, x_{9}\right) \in \mathbb{R}^{9}$ there is a one dimensional set of $\left(x_{10}, x_{11}, x_{12}\right) \in \mathbb{R}^{3}$ (namely those points on the ray $\left.R_{\text {out }}\left(s\left(x_{1}, x_{2}, x_{3}\right), t\left(x_{1}, x_{2}, x_{3}\right)\right)\right)$ so that $\left(x_{1}, x_{2}, x_{3}, \ldots, x_{12}\right) \in \mathcal{M}$. Thus $\mathcal{M}$ is $9+1=10$ dimensional.

To summarize, our problem has become reframed as finding a surface $\Gamma(s, t)$ which lies in $\mathcal{M}$ and whose tangent space is everywhere perpendicular to the vector fields $\widetilde{V}_{i}$. We propose to find such a surface that passes through an initial curve

$$
C(t)=\left(C_{1}(t), C_{2},(t), C_{3}(t), C_{4}(t), \ldots, C_{12}(t)\right)
$$

which lies in $\mathcal{M}$ and is perpendicular to the $\widetilde{V}_{i}$. It turns out these are precisely the dimensions and number of vector fields so that one expects unique solutions.

At this stage it is reasonable to mention some issues that arise is practice. For one, one does not want two mirrors to cross one another. Thus we will stay away from the part of $\mathbb{R}^{12}$ where this happens. In particular, we only care about points where the $\tilde{V}_{i}$ are defined. (Note they are linearly independent at $x$ if all are defined at $x$.) Another issue that might show up is that a ray path might unintentionally intersect one of the mirrors (perhaps even the mirror it just intentionally hit). The model ignores this problem. One can often avoid 
this problem with a careful choice of initial curve. We will proceed under the assumption that this does not happen.

In what follows next we make use of differential forms. The reader who would prefer a presentation in terms of matrices should see the appendix to [6] for a description of how to convert from one viewpoint to the other. It may be be adequate though for the reader to consider a concise summary here: Below when $\theta_{i}(V)$ is written it means the dot product of a certain vector with $V$ and that $d \theta_{i}(V, W)$ corresponds to an expression $V^{t} \mathbf{A} W$ for a certain matrix A. The main geometric idea is to use these equations to determine the tangent space to the surface in $\mathbb{R}^{12}$, and then use that information to create a slight extension of the surface (on the tangent planes), and repeat this process, creating a sort of family of extensions beginning with the initial curve. These curves lie on a surface which can be projected into four different 3-dimensional subspaces to get the four different solution surfaces. Thus, in a sense, the four different solution surfaces are "shadows" of a single surface in $\mathbb{R}^{12}$.

We consider the one-forms $\theta_{i}$ that are dual to the $\widetilde{V}_{i}$. That is, at a point in $\mathbb{R}^{12}, \theta_{i}$ is the linear map from tangent vectors $X$ defined by $\theta_{i}(X)=\left\langle\widetilde{V}_{i}, X\right\rangle$. We let $E$ be the 8dimensional distribution in $\mathbb{R}^{12}$ which is the joint kernel of these four one forms. That is, at each point $x \in \mathbb{R}^{12}, E(x)$ is the subspace of tangent vectors at $x$ which are perpendicular to the 4 dimensional subspace spanned by the 4 vectors $\tilde{V}_{i}(x)$ and hence $E(x)$ is 8 dimensional.

A vector $V$ with base point in $\mathcal{M}$ and lying in $E$ is called Kähler-regular if the set of 8 linear equations for $W$

$$
\begin{gathered}
\theta_{i}(W)=\theta_{2}(W)=\theta_{3}(W)=\theta_{4}(W)=0 \\
d \theta_{1}(V, W)=d \theta_{2}(V, W)=d \theta_{3}(V, W)=d \theta_{4}(V, W)=0
\end{gathered}
$$

has maximal rank $=8$, hence the solution space (as the kernel of a 12 by 8 matrix of rank $8)$ is 4-dimensional. In addition we insist that this 4-dimensional solution space intersects the 10-dimensional tangent space to $\mathcal{M}$ in a 2-dimensional (as expected) subspace $T^{2}(V)$. This is a generic condition for such $V$.

Since $\theta_{i}$ will be zero on any tangent vector to a solution surface $\Gamma, d \theta_{i}(X, Y)=0$ for any two tangent vectors $X$ and $Y$ to $\Gamma$. Thus if $V$ is tangent to $\Gamma$ at $\Gamma(s, t)$ and is Kähler-regular then the tangent space to $\Gamma$ at $\Gamma(s, t)$ must be $T^{2}(V)$. 


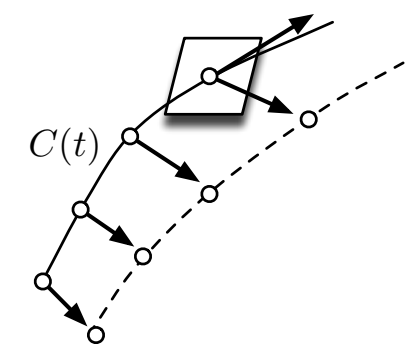

Fig. 3. Given a curve on our surface we use the known equations that it must satisfy to compute the tangent space at a discrete set of points on the curve, and then extend the surface discretely by moving out infinitesimally on the tangent spaces.

If all the data are reasonable, and if we start with an reasonable initial curve $C(t)$ (where reasonable means that they are real analytic) such that $C^{\prime}(t)$ is Kähler-regular then the Cartan-Kähler theorem (page 254 of [24]) guarantees that there exists a unique (locally defined) analytic surface $\Gamma$ solving our problem.

In practice (including the non analytic case), if we start with a Kähler-regular initial curve we can compute the tangent space to the solution surface and use that to get a nearby curve on the surface. The new curve should be approximately Kähler-regular so we can iterate and (at least for a while) generate more curves on the surface. We of course work with discrete approximations of the curves, and at each step generate a new discrete curve by moving out on the tangent space by a small amount, as is schematically depicted in Fig. 3. In the next section we give explicit examples.

\section{Examples}

In this section we describe some examples and present the results. In our first examples assume that the incoming bundle is collimated and has direction $(1,0,0)$, i.e. parallel to the $x$-axis, and the outgoing beam is a collimated beam with direction $(1,1,1)$. We create different cases by varying parameters of the problem. Our last two examples produce output bundles which are portions of bundles that pass through a point.

We choose a parameter $r$ which may expand $(r>1)$ or contract $(r<1)$ the ray bundle. We also choose how to orient it, i.e. twist the bundle. We do this by choosing two orthogonal unit vectors $Y_{\text {out }}$ and $Z_{\text {out }}$ and then for each $(s, t)$ (near $(0,0)$ ) we choose $R_{\text {out }}(s, t)$ to be the 
ray in the direction $(1,1,1)$ that passes through the point $\left(x_{0}, y_{0}, z_{0}\right)+r s Y_{\text {out }}+r t Z_{\text {out }}$. Here we choose the point $\left(x_{0}, y_{0}, z_{0}\right)$ rather arbitrarily (though it must be away from $(0,0,0)$ ) to be $(40,10,0)$. We will give examples for various values of $r$ and two different choices of $Y_{\text {out }}$ and $Z_{\text {out }}$. To begin we take $r=3$ (i.e. we expand the beam by a factor of 3 ) with $Y_{\text {out }}=\frac{1}{\sqrt{6}}(-2,1,1)$ and $Z_{\text {out }}=\frac{1}{\sqrt{2}}(0,-1,1)$.

In general, the success of the numerical method is sensitive to choice of initial conditions. We now describe a choice of initial curve that yielded some good results. The initial curve in $\mathbb{R}^{12}$ consists of four separate parameterized curves in 3 -space. We choose the first two and the last one to be straight lines as follows:

$$
\left(0, t, 0,0, t, 10+0.05 t, C_{7}(t), C_{8}(t), C_{9}(t), 40+r Y_{\text {out }}(1) t, 10+r Y_{\text {out }}(2) t, r Y_{\text {out }}(3) t\right) .
$$

(The 0.05 in the sixth slot is some arbitrary small nonzero constant - its choice can affect the results some.) Observe that the above choices guarantee that the initial curve will lie in $\mathcal{M}$. The other three components are determined by the fact that $\theta_{2}, \theta_{3}$, and $\theta_{4}$ are zero on the initial curves. This gives us two algebraic equations and one first-order ordinary differential equation to solve. Thus there is one more parameter to choose - the initial condition of this differential equation. This allows us to choose $C_{9}(0)$ (i.e. the initial z value of the third curve). Here we choose this to be 15. In the top of Fig. 4 we see such an initial curve (i.e. four parameterized curves). The curves in numbered in Fig. 4 from 1-4, with curve 3 being the one that must be solved for. In the lower half of Fig. 4 we see the paths taken by the light rays between the curves. The incoming and outgoing rays are not shown in the figure.

Now that we have chosen a good initial curve we may generate the four surfaces. In Fig. 5 we see the four surfaces in their relative positions, although not plotted to scale.

We now address the question as to how one can graphically test our designs. Assuming that one has an imaging system with the described reflectors, we consider a test pattern, which is a photograph of a parking lot (see Fig. 6). We image this pattern to the plane $x=-100$ using the orthographic projection induced by the four mirror system (since it maps a collimated bundle to a collimated bundle) and observe how the reflectors change the image that is formed and how the image changes with the placement of the test pattern. With respect to Fig. 4, the arrow in that figure is pointed in the opposite direction of what would be the physical path of the light in our simulated imaging system.

We consider three separate simulations with the test pattern centered at $(240,210,200)$, 

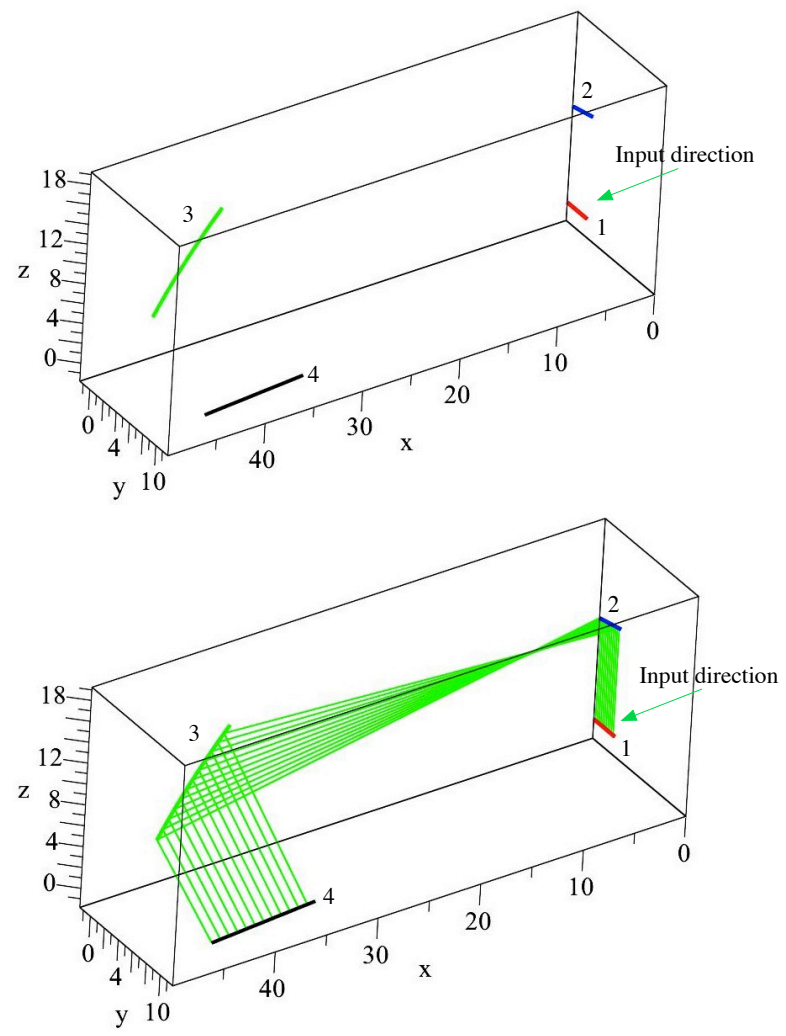

Fig. 4. The above figure depicts the four initial curves in 3-space and below that we see the trajectories of rays.

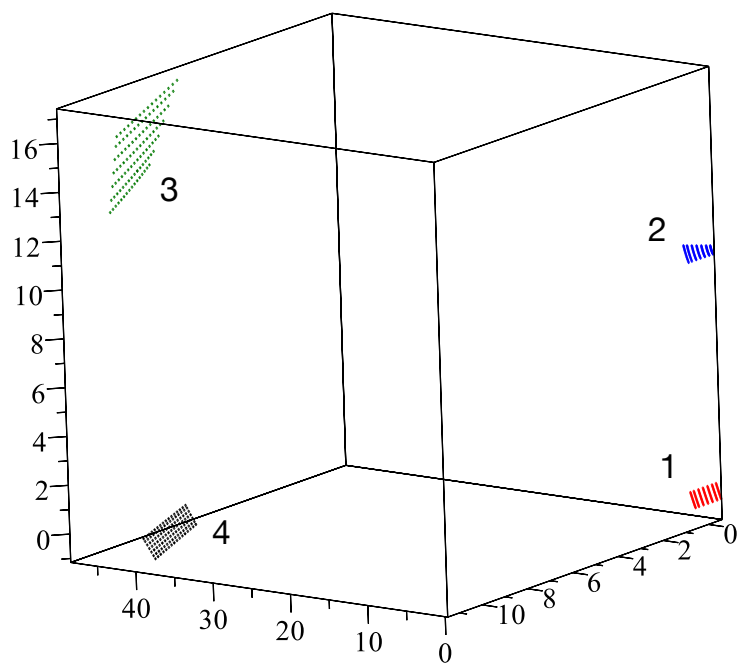

Fig. 5. The four reflector system (not plotted to scale), numbered as in Fig. 4. 


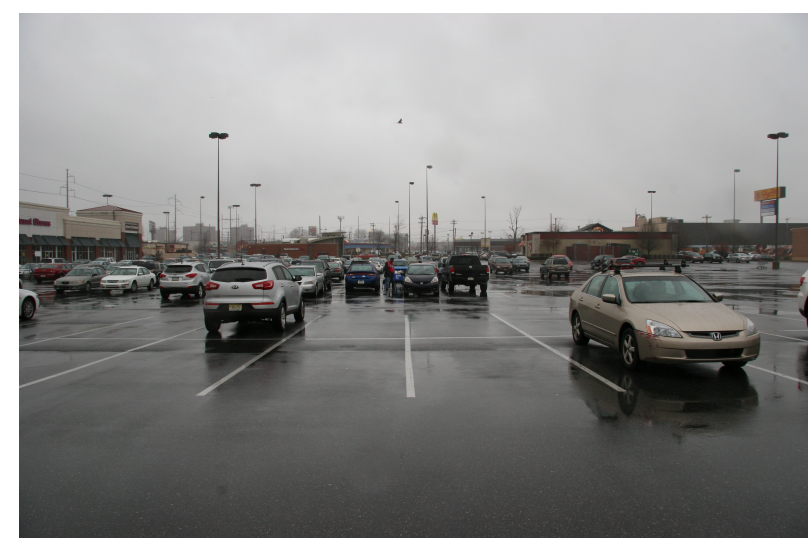

Fig. 6. The test pattern (image) used in the test

$(640,610,600)$, and $(940,910,900)$, and perpendicular to the $(1,1,1)$ direction - i.e. along the output ray $R_{\text {out }}(0,0)$. If our design theory and numerics are correct we should see a rotated portion of the test pattern. The size (i.e. fraction of the pattern) we see is proportional to $r$. Since the output beam should be collimated we should get exactly the same result for all three trials, despite the increase in distance to the test pattern. Fig. 7 depicts the results, which are consistent with our model. In general this is how we tested if our mirror systems preserved collimation, and we always found in all of our examples that collimation was well preserved.

Variants of this example where $r=5$ and $r=10$ with the same rotation of the bundle (i.e. same $Y_{\text {out }}$ and $Z_{\text {out }}$ ) appear in Fig. 8 and Fig. 9. In the case of $r=10$ the test pattern does not fill the entire reflector, and we see the checkerboard test pattern behind it.

In another example, we again take $r=3$, but let $Y_{\text {out }}=\frac{1}{\sqrt{2}}(-1,1,0)$ and $Z_{\text {out }}=$ $\frac{1}{\sqrt{1.5}}(-0.5,-0.5,1)$ so that the bundle is not twisted and as a result the test pattern does not appear rotated. The results for two different placements of the test pattern appear in Fig. 10. In the the bottom image of Fig. 10 we see for the first time some problems in the image quality. Here we suspect we are seeing either artifacts of the numerical method for solving the partial differential equations, not due to the ray tracer. We do not pursue this point further here, but include it to demonstrate that there are subtle numerical issues that need to be handled sometimes regarding the solution scheme and the data structures that represent a smooth surface.

Fig. 11 depicts results in the case of $r=0.5$, hence obtaining a narrower output bundle than the incoming bundle. 

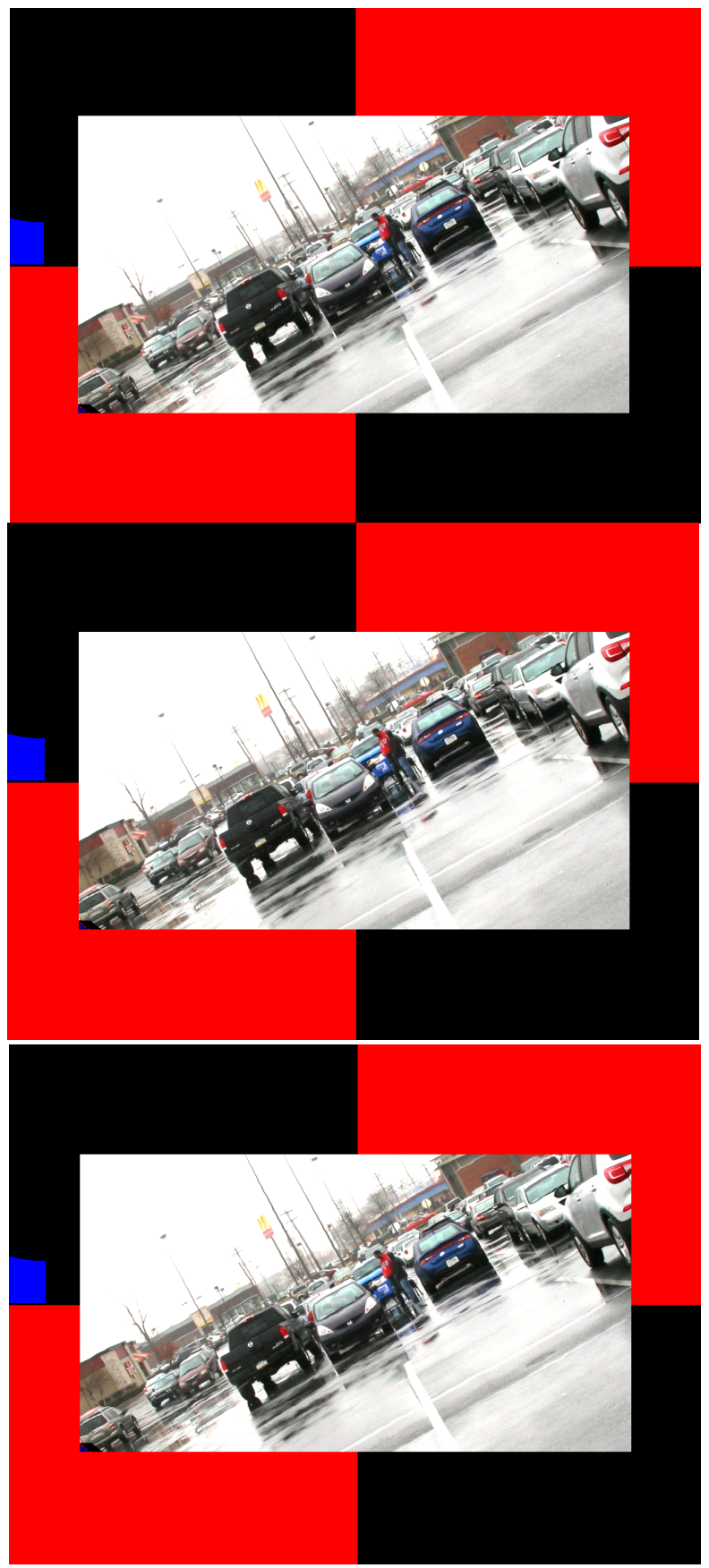

Fig. 7. Three tests with the test pattern at $(240,210,200),(640,610,600)$, and $(940,910,900)$ respectively. They should be very similar due to the collimation of the output beam. Note that the mirrors are simulated in a virtual room with checkerboard walls of various colors and some sample text. 


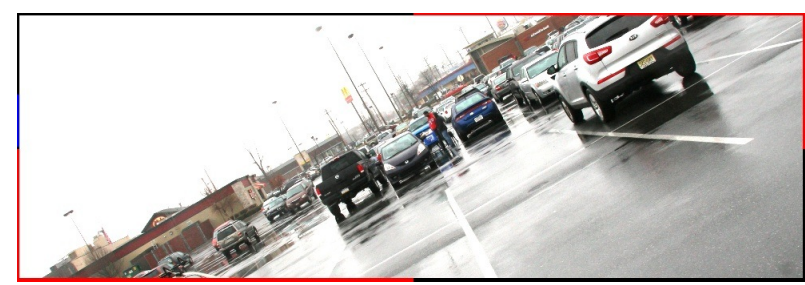

Fig. 8. An image of the test pattern via rotated collimated bundle with $r=5$.

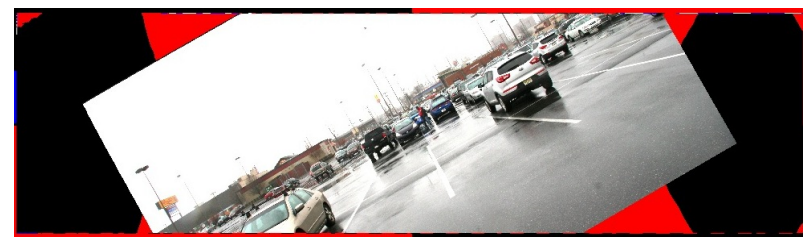

Fig. 9. An image of the test pattern via a rotated collimated bundle with $r=10$.

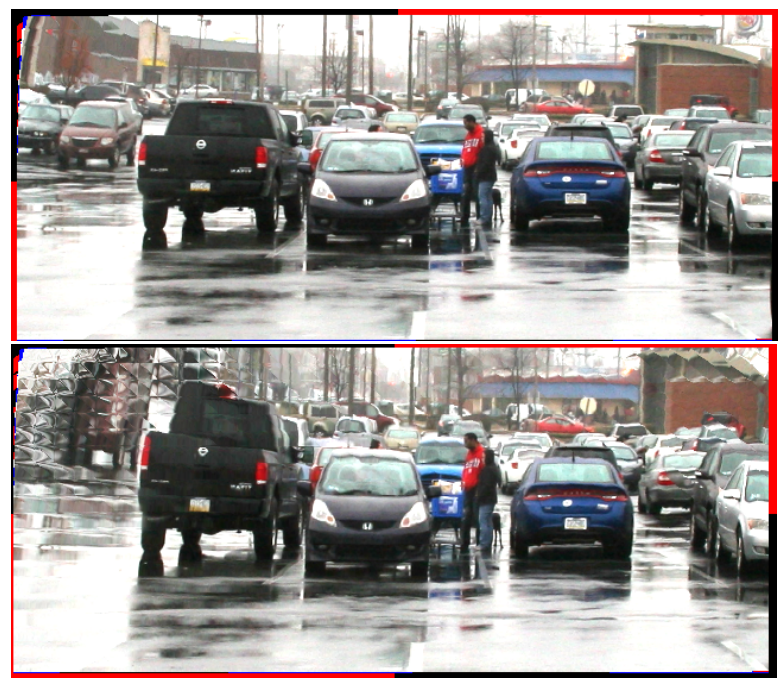

Fig. 10. Images of the test pattern (placed at different positions) via collimated bundle with $r=3$ and no twist. We can see from from the distortion in the lower image that the bundle is not perfectly collimated.

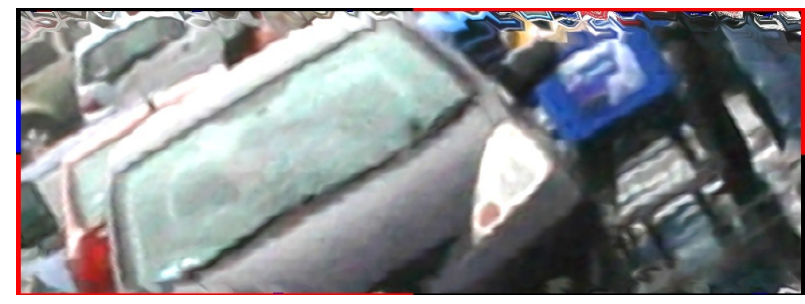

Fig. 11. An image of the test pattern via rotated collimated bundle with $r=0.5$. 

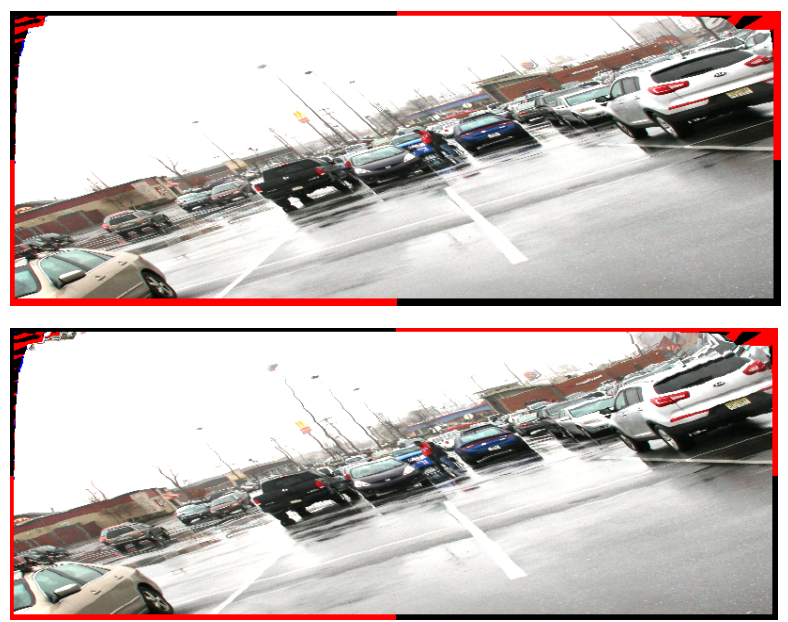

Fig. 12. Here the $y$ scaling is 4 and $z$ scaling is 8 with the image plane at $(240,210,200)$ (top) and $(940,910,900)$ (bottom).

If we scale the $y$ direction by 4 and the $z$ direction by 8 before twisting the bundle, then one obtains the images depicted in Fig. 12.

Finally, we present two examples where the output beam is not collimated. Both have the output rays that all pass through a single virtual point. The central output ray in both cases still points in the $(1,1,1)$ direction and passes through $(40,10,0)$. The virtual point in the first case is $(40,10,0)-\frac{20}{\sqrt{3}}(1,1,1)$ while in the second case it is at $(40,10,0)+\frac{700}{\sqrt{3}}(1,1,1)$.

In the first case the output beam seems to emanate from a point source at the virtual point. We show a sequence of POVRay trials with the test pattern (which is twice as big as in previous trials) centered at various points along the central output ray in Fig. 13. We should (and do) get images where the test pattern appears progressively smaller (i.e. we see imaging a progressively portion of it).

In the second case the output beam should focus to a point (approximately $(444,414,404)$ ) and then invert through that point. The sequence of images from POVRay tests should see progressively smaller pieces of the test pattern until $(444,414,404)$ and then the images should invert the test pattern and see more and more of it. We see a sequence of such results in Fig. 14. An image depicting the catastrophic nature of passing through the focus is included. 


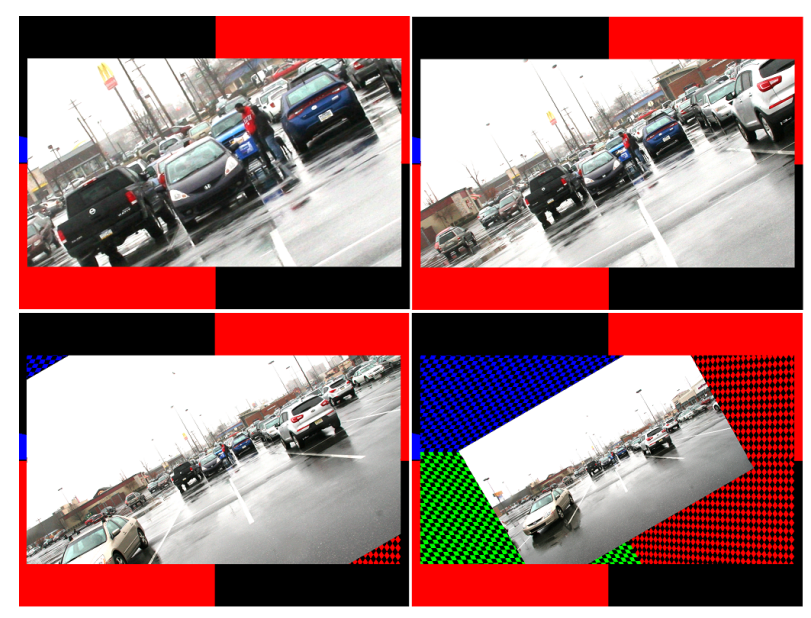

Fig. 13. From top left to bottom right: taking $r=3$, a series of POVRay tests with the test pattern centered at $(140,110,100),(240,210,200),(640,610,600)$ and $(840,810,800)$ respectively for the output beam from virtual point source at $(40,10,0)-\frac{20}{\sqrt{3}}(1,1,1)$.

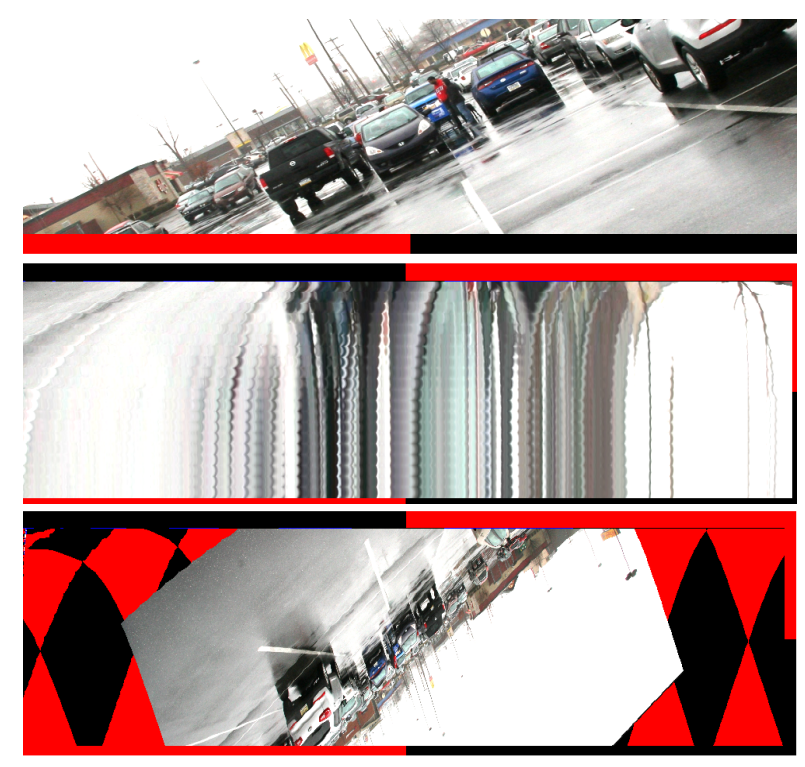

Fig. 14. From top to bottom: taking $r=3$, a series of POVRay tests with the test pattern centered at $(140,110,100),(440,410,400)$, which is near the focal point, and $(940,910,900)$ respectively for the output beam from virtual point source at $(40,10,0)+\frac{700}{\sqrt{3}}(1,1,1)$.

\section{Conclusions}

We have described a theoretical framework for the control of a single ray bundle based on the theory of EDS. In earlier work we had used EDS as a basis of a design technique for two 
reflector systems that controlled a single ray bundle to a lesser extent. Here, using simulated four reflector systems, we performed numerical experiments that demonstrate not only that the ray bundle passes through the required points, but also in the required directions.

\section{Acknowledgements}

Chris Croke was supported in part by NSF grant DMS 10-03679 and an Eisenbud Profes-

sorship at the Mathematical Sciences Research Institute. R. Andrew Hicks was supported in part by NSF grant DMS 09-08299. 


\section{References}

[1] R. Andrew Hicks and Ruzena Bajcsy. Catadioptic sensors that approximate wide-angle perspective projections. In Proc. Computer Vision Pattern Recognition, pages 545-551. IEEE Computer Society, Los Alamitos, 2000.

[2] D. G. Burkhard and D. L. Shealy. Design of reflectors which will distribute sunlight in a specified manner. Journal of Solar Energy, 17:221-227, 1975.

[3] J.H. McDermit and T.E. Horton. Reflective optics for obtaining prescribed irradiative distributions from collimated sources. Applied Optics, 13:1444-1450, 1974.

[4] R. Andrew Hicks and R. Perline. The blind-spot problem for motor vehicles. Applied Optics, 44:3893-3897, 2005.

[5] R. Andrew Hicks. Controlling a ray bundle with a free-form reflector. Opt. Lett., 33(15):16721674, 2008.

[6] R. Andrew Hicks and Christopher Croke. Designing coupled free-form surfaces. J. Opt. Soc. Am. A, 27(10):2132-2137, Oct 2010.

[7] Sergey A. Kochengin and Vladimir I. Oliker. Determination of reflector surfaces from near-field scattering data. Inverse Problems, 13(2):363-373, 1997.

[8] S. Kochengin and V. Oliker. Determination of reflector surfaces from near-field scattering data. II. Numerical solution. Numer. Math., 79(4):553-568, 1998.

[9] J. S. Schruben. Formulation of a reflector-design problem for a lighting fixture. J. Opt. Soc. Am., 62(12):1498-1501, Dec 1972.

[10] C.W. Kanolt. Multifocal Opthalmic Lenses. United States Patent 2,878,721, March 24th, 1959.

[11] William T. Plummer. Unusual optics of the polaroid sx-70 land camera. Appl. Opt., 21(2):196208, Jan 1982.

[12] E. H. Land. Reflex camera, June 27 1972. US Patent 3,672,281.

[13] J. G. Baker. Compact folding reflex camera, July 25 1972. US Patent 3,678,831.

[14] W. T. Plummer. Reflective imaging apparatus, May 29 1973. US Patent 3,735,685.

[15] E. Kreifeldt. Darpa turns researchers loose on new class of optics. Optics and photonics News, 8(1):6-10, 1997.

[16] Robert R Shannon, James P Mills, Harvey M Pollicove, Patrick A Trotta, and LN Durvasula. Optics that fit. Photonics Spectra, 97:86-88, 2001. 
[17] Bryan D. Stone and G. W. Forbes. Foundations of first-order layout for asymmetric systems: an application of hamilton's methods. J. Opt. Soc. Am. A, 9(1):96-109, Jan 1992.

[18] Bryan D. Stone and G. W. Forbes. Characterization of first-order optical properties for asymmetric systems. J. Opt. Soc. Am. A, 9(3):478-489, Mar 1992.

[19] Jacob Rubinstein and Gershon Wolansky. Intensity control with a free-form lens. J. Opt. Soc. Am. A, 24(2):463-469, Feb 2007.

[20] R. Winston, J. Minano, and P. Benitez. Nonimaging Optics. Elsevier Academic Press, Burlington, Mass., 2005.

[21] David L. Shealy. Theory of geometrical methods for design of laser beam shaping systems. Proc. SPIE, 4095:1-15, 2000.

[22] R. Winston. Selected Papers on Nonimaging Optics. SPIE Optical Engineering Press, Bellingham, Wash., 1995.

[23] W.T. Welford and R. Winston. The optics of nonimaging concentrators: light and solar energy. Academic Press, San Diego, CA, 1978.

[24] T. Ivey and J.M. Landsberg. Cartan for Beginners: Differential Geometry via Moving Frames and Exterior Differential Systems. American Mathematical Society, Providence Rhode Island, 2003. 